\title{
Thyroid autoimmunity in children and adolescents with newly diagnosed type 1 diabetes mellitus
}

\author{
Eui Seok Jung, MD', \\ Dong Kyun Han, MD', \\ Eun Mi Yang, MD', \\ Min Sun Kim, MD', \\ Dae-Yeol Lee, $\mathrm{MD}^{2}$, \\ Chan Jong Kim, MD' \\ ${ }^{1}$ Department of Pediatrics, \\ Chonnam National University \\ Medical School, Gwangju \\ ${ }^{2}$ Department of Pediatrics, \\ Chonbuk National University \\ Medical School, Jeonju, Korea
}

Received: 22 April, 2014

Revised: 16 June, 2014

Accepted: 20 June, 2014

Address for correspondence: Chan Jong Kim, MD

Department of Pediatrics, Chonnam National University Hospital \& Medical School, 42 Jebong-ro, Dong-gu, Gwangju 501-757, Korea Tel: +82-62-220-6645

Fax: +82-62-222-6103

E-mail: cjkim@jnu.ac.kr
Purpose: This study aim to investigate the occurrence of autoimmune thyroid disease in children and adolescents at onset of type 1 diabetes mellitus (T1DM) and to assess whether the presence of diabetes-specific autoantibodies can predict the autoimmune thyroid disorder.

Methods: Seventy-three children with T1DM were recruited. Glutamic acid decarboxylase antibodies (GADA), islet cell antibodies (ICA), insulin autoantibodies (IAA), and thyroid antibodies were determined in all patients at the time of diagnosis.

PResults: The majority of patients (87.7\%) had at least one pancreatic antibody (74.0\% for GADA, $20.5 \%$ for ICA, and $24.7 \%$ for IAA). Thyroid autoantibodies were found in 19 of 73 patients $(26.0 \%)$ at diagnosis. Thyroid autoimmunity (TA) incidence was not statistically significant by GADA or ICA positivity, but significantly higher by IAA positivity $(P=0.03)$, and IAA positivity showed odds ratio, $4.931 ; 95 \%$ confidence interval, 1.323-18.381 for TA.

Conclusion: The IAA positivity in children and adolescents with TIDM was strongly related to positivity of thyroid autoantibodies and thus it could serve as an index for early prediction of the development of the thyroid autoimmune disorder among children and adolescents with TIDM.

Keywords: Type 1 diabetes mellitus, Thyroiditis; Autoimmunity

\section{Introduction}

Type 1 diabetes mellitus (T1DM) is a common endocrine disorder associated with aberrant immune responses to specific $\beta$-cell autoantigens ${ }^{1,2)}$. Markers of the process are various including autoantibodies to glutamic acid decarboxylase (GADA), to islet cell (ICA) and to insulin (IAA) ${ }^{3)}$. These patients are also prone to other autoimmune disorders such as Graves' disease, Hashimoto's thyroiditis, Addison's disease, vitiligo, celiac sprue, autoimmune hepatitis, myasthenia gravis, and pernicious anemia ${ }^{4}$.

The most prevalent autoimmune disease associated with T1DM is autoimmune thyroid disorder, characterized by the presence of thyroid antibodies to peroxidase and thyroglobulin. The prevalence of positive thyroid antibodies in children with T1DM is $~ 50 \%$ in different countries but varies with age, sex, and ethnic origin of patients ${ }^{5)}$. Thyroid diseases occur within 3 to 4 years in half of the thyroid antibody positive groups ${ }^{6,7)}$. It is inferred that TIDM and autoimmune thyroiditis are based on a common genetic origin, as they have similar pathogenicity and frequently occur simultaneously in the same individual and/or the same family ${ }^{8}$. Some pancreatic antibodies, known to be related to autoimmune diseases, are specially associated with an autoimmune reaction to thyroid antigens ${ }^{9,10)}$.

The aim of the present study was to investigate the occurrence of autoimmune thyroid disease in children and adolescents at the onset of T1DM and to assess whether the presence of diabetes-specific autoantibodies can predict thyroid autoimmunity (TA). 


\section{Materials and methods}

\section{Subjects}

The study was undertaken at the Chonnam National University Hospital and Chonbuk National University Hospital located in the Honam region of Korea. We retrospectively analyzed the medical records from children and adolescents who had been diagnosed with T1DM between January 2007 and June 2011. All patients met the criteria of American Diabetes Association for TIDM $^{4)}$. Patients who were not tested for all pancreatic autoantibodies and thyroid antibodies were excluded. We reviewed the demographic data, history of diabetes onset, and thyroid palpation findings. This study was approved by the Institutional Review Board of each hospital. The recommendations of the Declaration of Helsinki for biomedical research involving human subjects were followed.

\section{Methods}

Levels of blood glucose and glycosylated hemoglobin (HbAlc) were assessed and GADA, ICA, and IAA were evaluated at the time of diagnosis. Screening for TA was performed using measurements of thyroid antiperoxidase antibody (TPOAb), antithyroglobulin antibody (TGAb), and thyroid stimulating hormone (TSH) receptor antibodies (TSHRAb). Free thyroxine and TSH were also measured. TA was defined as the presence of at least one thyroid autoantibody. GADA was assessed by radioimmunoassay kit (RSR Ltd., Cardiff, UK), IAA by enzyme immunoassay kit (Orgentec Diagnostika, Mainz, Germany), and ICA by the indirect fluorescent antibody technique (SCIMEDX, Denville, NJ, USA). TPOAb (normal range, $0-34 \mathrm{IU} / \mathrm{mL}$ ) and TGAb (normal range, $0-115 \mathrm{IU} / \mathrm{mL}$ ) were measured using electrochemiluminiscence immunoassay kit (COBAS, Roche Diagnostics GmbH, Mannheim, Germany) and TSHRAb (normal range, $0-15 \mathrm{IU} / \mathrm{L}$ ) was determined by radioimmunoassay (RSR Ltd.).

\section{Statistical analysis}

Data are expressed as mean \pm standard deviation or proportion (\%). The unpaired Student $t$-test was performed to compare continuous variables, and the chi-square test was used to compare percentages among different patient subgroups. Multiple logistic regression analysis was used to assess the strength and independency of the associations. Outcomes included pancreatic antibody positivity and TA. All statistical analyses were performed with IBM SPSS ver. 20.0 (IBM Co., Armonk, NY, USA). A $P$-value $<0.05$ was considered significant.

\section{Results}

Of the 98 patients selected for the study, 25 patients who were not tested for all pancreatic autoantibodies and thyroid antibodies were excluded. Therefore, 73 patients were studied. Of the 73 patients, 30 were boys (41.1\%) and 43 (58.9\%) were girls. The mean age at diagnosis of diabetes was $8.3 \pm 3.7$ years. Mean HbA1c at onset of diabetes was $12.0 \% \pm 2.1 \%$ and body mass index was $15.6 \pm 3.2 \mathrm{~kg} / \mathrm{m}^{2}$. The majority of patients $(64$ of $73,87.7 \%$ ) had at least one pancreatic antibody at onset of TIDM, whereas only nine patients (12.3\%) were negative for all three antibodies. In particular, 54 patients $(74.0 \%)$ were positive for GADA, 15 (20.5\%) for ICA, and 18 (24.7\%) for IAA, respectively (Table 1). Only one antibody was present in 44 patients $(60.3 \%), 17$ patients $(23.3 \%)$ were positive for two of three, and three $(4 \%)$ for all three antibodies. Nineteen patients (26.0\%) were positive for thyroid autoantibody at diagnosis of TIDM. 73.7\% of patients with positive thyroid antibodies were female compared with $53.7 \%$ in patients without antibodies.

All 19 patients in the thyroid antibody positive group were positive for at least one pancreatic autoantibody (100\% vs. $83.3 \%, P=0.01$ ) (Table 1). Of the 19 patients with TA, 13 (68.4\%) patients were positive for GADA, $5(26.3 \%)$ were positive for ICA. And nine of 19 (47.4\%) were significantly positive for IAA $(P=0.03)$. The prevalence of TPOAb, TGAb, and TSHRAb was $15.1 \%, 21.9 \%$, and $2.7 \%$ in patients with T1DM, respectively. Only 1 antibody was present in 10 patients (13.7\%), eight patients (11.0\%) were positive for two of three, and one $(1.3 \%)$

Table 1. Clinical features of type 1 diabetes patients with and without thyroid antibodies

\begin{tabular}{|c|c|c|c|c|}
\hline Variable & All patients $(n=73)$ & Thyroid Ab positive $(n=19)$ & Thyroid Ab negative $(n=54)$ & $P$-value \\
\hline Sex (M:F) & $30: 43$ & $5: 14$ & $25: 29$ & 0.12 \\
\hline Age at diagnosis (yr) & $8.3 \pm 3.7$ & $7.7 \pm 4.1$ & $8.4 \pm 3.5$ & 0.94 \\
\hline HbA1c at diagnosis (\%) & $12.0 \pm 2.1$ & $11.9 \pm 2.5$ & $12.0 \pm 2.0$ & 0.88 \\
\hline DKA at diagnosis (\%) & $24(32.8)$ & $7(36.8)$ & $17(31.5)$ & 0.67 \\
\hline Body mass index $\left(\mathrm{kg} / \mathrm{m}^{2}\right)$ & $15.6 \pm 3.2$ & $15.8 \pm 2.4$ & $15.5 \pm 3.5$ & 0.77 \\
\hline Pancreatic Ab positive (\%) & $64(87.7)$ & 19 (100) & $45(83.3)$ & 0.01 \\
\hline GADA positive (\%) & $54(74.0)$ & $13(68.4)$ & $41(75.9)$ & 0.53 \\
\hline ICA positive (\%) & $15(20.5)$ & $5(26.3)$ & $10(18.5)$ & 0.48 \\
\hline IAA positive (\%) & $18(24.7)$ & $9(47.4)$ & $9(16.7)$ & 0.03 \\
\hline
\end{tabular}

Values are presented as mean \pm standard deviation or number (\%).

Ab, antibody; HbA1c, glycosylated hemoglobin; DKA, diabetic ketoacidosis; GADA, glutamic acid decarboxylase antibody; ICA, islet cell autoantibody; IAA, insulin autoantibody. 
for all three antibodies.

The prevalence rates of thyroid autoantibodies are shown according to the presence of each pancreatic antibody (Table 2). The prevalence of overall TA was as follows; $13 / 54$ (24.1\%) in the GADA positive group, 5/15 (33.3\%) in the ICA positive group, and $9 / 18(50 \%)$ in the IAA positive group, respectively. The prevalence of TA was not significantly influenced by GADA or ICA positivity, but was significantly increased by IAA positivity (odds ratio, 4.931; 95\% confidence interval, 1.323-18.381) (Table 3).

\section{Discussion}

T1DM is an autoimmune disease in most cases. The incidence of T1DM varies widely across the world, from the highest incidence in northern Europe to the lowest incidence in Asia ${ }^{11)}$. T1DM is frequently associated with other autoimmune disorders, particularly endocrinological diseases ${ }^{7,8,12,13)}$. Autoimmune thyroid disorders are the most prevalent immunological diseases in patients with $\mathrm{T} 1 \mathrm{DM}^{7,8,12)}$. Autoimmune destruction of the thyroid gland may cause either hypothyroidism or hyperthyroidism. We identified autoantibodies to islet cells and the thyroid in a number of Korean children and adolescents with TIDM. Substantial variations in the prevalence of autoantibodies have been reported in other ethnic groups. The prevalence rates of antibodies in Japanese patients with TIDM are 60\%-70\% for GADA, $45 \%-50 \%$ for IAA, and $60 \%-65 \%$ for IA- 2 autoantibodies at disease onset, which are similar to those reported in Caucasian patients ${ }^{14)}$. In our study, 64 patients (87.7\%) had at least one pancreatic antibody (GADA, 74.0\%; ICA, 20.5\%; and IAA, 24.7\%).

The prevalence of thyroid dysfunction increases with the presence of thyroid autoantibodies. TPOAb and/or TGAb can be detected long before changes in TSH and thyroid hormone levels occur ${ }^{5,7,8}$. Thus determining these antibody levels might be useful for early diagnosis of the disease before thyroid dysfunction develops. The prevalence of thyroid autoantibodies in children with T1DM varies widely between $3 \%$ and $50 \%$ according to different studies ${ }^{5,7,8,13,15)}$. It has been suggested that the coexistence of thyroid dysfunction might cause disturbances in glycemic control of patients with diabetes ${ }^{16}$. Even subclinical hypothyroidism (slightly elevated TSH without impairment of T4 and T3 levels) is associated with a higher frequency of symptomatic hypoglycemia ${ }^{17}$. Furthermore, Chase et al. ${ }^{18)}$ reported a reduced rate of growth in diabetic children with elevated TSH values and thyromegaly, but normal serum thyroxine levels. Treatment with L-thyroxine leads to significantly improved growth in prepubertal children compared with age-matched diabetic controls. Thyroid function tests and ultrasound assessments are recommended in cases of antibody positivity to minimize the risk of undiagnosed hypothyroidism in young patients with diabetes. From our study, the prevalence rates of TPOAb, TGAb, and TSHRAb were $15.1 \%, 21.9 \%$, and $2.7 \%$ in patients with T1DM, respectively. The overall prevalence of TA was $26.0 \%$, which was similar to the findings in other studies. In Germany and Austria, Kordonouri et al. ${ }^{5,10)}$ reported a prevalence of $21 \% \mathrm{TA}$, whereas a prevalence of $50 \%$ has been reported in a Caucasian population with diabetes in the USA ${ }^{6}$. Other Asian studies have reported a prevalence of $21.8 \%$ in a Taiwanese population and $18 \%$ in a Japanese population ${ }^{19,20)}$.

Jin et al." found that thyroid antibody-positive TIDM patients have a higher frequency of GADA than thyroid antibodynegative patients and that a high titer of GADA is a strong predictor for the development of TA in patients with TIDM. A possible explanation for this association could be that GAD is not exclusively present in the pancreas but can also be found in other tissues, such as the follicle cells of the thyroid gland and the parietal cells of the stomach ${ }^{21,22}$. Thus, the persistence of GADA could be a marker for the future development of autoimmunity against the thyroid gland and other organs. Chang et al. ${ }^{19)}$ reported the absence of correlation between TPOAb and GADA in Taiwanese TIDM patients and suggested genetic heterogeneity in the role of autoimmunity of TIDM and autoimmune thyroid disease among races. In the present study, the prevalence of overall thyroid antibody positivity was as follows; 13 (24.1\%) in the GADA-positive group, five (33.3\%)

Table 3. Association between pancreatic antibodies and thyroid autoimmunity

\begin{tabular}{lcc}
\hline Factor & OR $(95 \% \mathrm{Cl})$ & $P$-value \\
\hline GADA positivity & $1.320(0.335-5.209)$ & 0.692 \\
ICA positivity & $1.140(0.300-4.325)$ & 0.848 \\
IAA positivity & $4.931(1.323-18.381)$ & 0.017 \\
\hline
\end{tabular}

OR, odds ratio; $\mathrm{Cl}$, confidence interval; GADA, glutamic acid decarboxylase antibody; ICA, islet cell autoantibody; IAA, insulin autoantibody.

Table 2. Prevalence of thyroid antibodies according to the presence of each pancreatic antibody

\begin{tabular}{lcccc}
\hline Variable & GADA positive $(n=54)$ & ICA positive $(n=15)$ & IAA positive $(n=18)$ & PA positive $(n=64)$ \\
\hline TPOAb positivity & $8(14.8)$ & $2(13.3)$ & $4(22.2)$ & $11(17.2)$ \\
TGAb positivity & $11(20.4)$ & $5(33.3)$ & $8(44.4)$ & $16(25.0)$ \\
TSHRAb positivity & $0(0)$ & $1(6.7)$ & $2(11.1)$ & $2(3.1)$ \\
TA positivity & $13(24.1)$ & $5(33.3)$ & $9(50.0)$ & $19(26.0)$ \\
\hline
\end{tabular}

Values are presented as number (\%).

GADA, glutamic acid decarboxylase antibody; ICA, islet cell autoantibody; IAA, insulin autoantibody; PA, pancreatic antibody; TPOAb, thyroid antiperoxidase antibody; TGAb, antithyroglobulin antibody; TSHRAb, thyroid stimulating hormone receptor antibody; TA, thyroid autoimmunity. 
in the ICA-positive group, and nine (50\%) in the IAA positive group, respectively. TA incidence was not statistically significant by GADA or ICA positivity but significantly higher by IAA positivity, and IAA positivity showed a odds ratio, 4.931; 95\% confidence interval, 1.323-18.381 for TA.

In conclusion, the prevalence of TA in our study was $26.0 \%$ in children and adolescents with TIDM. IAA positivity in children and adolescents with TIDM was strongly related to the positivity of thyroid autoantibodies and thus it could serve as an index for early prediction of the development of the thyroid autoimmune disorder among children and adolescents with TIDM. Long-term prospective investigations with large populations of individuals are needed to elucidate the natural history of autoimmunity in patients with TIDM.

\section{Conflict of interest}

No potential conflict of interest relevant to this article was reported.

\section{References}

1. Atkinson MA, Eisenbarth GS. Type 1 diabetes: new perspectives on disease pathogenesis and treatment. Lancet 2001;358:221-9.

2. Lindberg B, Ericsson UB, Ljung R, Ivarsson SA. High prevalence of thyroid autoantibodies at diagnosis of insulin-dependent diabetes mellitus in Swedish children. J Lab Clin Med 1997;130:585-9.

3. Kimpimäki T, Kulmala P, Savola K, Kupila A, Korhonen S, Simell T, et al. Natural history of beta-cell autoimmunity in young children with increased genetic susceptibility to type 1 diabetes recruited from the general population. J Clin Endocrinol Metab 2002;87:4572-9.

4. American Diabetes Association. Diagnosis and classification of diabetes mellitus. Diabetes Care 2011;34 Suppl 1:S62-9.

5. Kordonouri O, Klinghammer A, Lang EB, Gruters-Kieslich A, Grabert M, Holl RW. Thyroid autoimmunity in children and adolescents with type 1 diabetes: a multicenter survey. Diabetes Care 2002;25:1346-50.

6. Burek CL, Rose NR, Guire KE, Hoffman WH. Thyroid autoantibodies in black and in white children and adolescents with type 1 diabetes mellitus and their first degree relatives. Autoimmunity 1990;7:157-67.

7. Umpierrez GE, Latif KA, Murphy MB, Lambeth HC, Stentz F, Bush A, et al. Thyroid dysfunction in patients with type 1 diabetes: a longitudinal study. Diabetes Care 2003;26:11815.

8. Mantovani RM, Mantovani LM, Dias VM. Thyroid autoimmunity in children and adolescents with type 1 diabetes mellitus: prevalence and risk factors. J Pediatr Endocrinol Metab 2007;20:669-75.
9. Jin P, Huang G, Lin J, Yang L, Xiang B, Zhou W, et al. High titre of antiglutamic acid decarboxylase autoantibody is a strong predictor of the development of thyroid autoimmunity in patients with type 1 diabetes and latent autoimmune diabetes in adults. Clin Endocrinol (Oxf) 2011;74:587-92.

10. Kordonouri O, Charpentier N, Hartmann R. GADA positivity at onset of type 1 diabetes is a risk factor for the development of autoimmune thyroiditis. Pediatr Diabetes 2011;12:31-3.

11. Karvonen M, Viik-Kajander M, Moltchanova E, Libman I, LaPorte R, Tuomilehto J. Incidence of childhood type 1 diabetes worldwide. Diabetes Mondiale (DiaMond) Project Group. Diabetes Care 2000;23:1516-26.

12. Levy-Shraga Y, Lerner-Geva L, Boyko V, Graph-Barel C, Mazor-Aronovitch K, Modan-Moses D, et al. Type 1 diabetes in pre-school children: long-term metabolic control, associated autoimmunity and complications. Diabet Med 2012;29:1291-6.

13. Radetti G, Paganini C, Gentili L, Bernasconi S, Betterle C, Borkenstein M, et al. Frequency of Hashimoto's thyroiditis in children with type 1 diabetes mellitus. Acta Diabetol 1995;32:121-4.

14. Kawasaki E, Eguchi K. Is Type 1 diabetes in the Japanese population the same as among Caucasians? Ann N Y Acad Sci 2004; 1037:96-103.

15. Kordonouri O, Deiss D, Danne T, Dorow A, Bassir C, Gruters-Kieslich A. Predictivity of thyroid autoantibodies for the development of thyroid disorders in children and adolescents with Type 1 diabetes. Diabet Med 2002;19:51821.

16. Kordonouri O, Maguire AM, Knip M, Schober E, Lorini R, Holl RW, et al. Other complications and associated conditions with diabetes in children and adolescents. Pediatr Diabetes 2009;10 Suppl 12:204-10.

17. Mohn A, Di Michele S, Di Luzio R, Tumini S, Chiarelli F. The effect of subclinical hypothyroidism on metabolic control in children and adolescents with Type 1 diabetes mellitus. Diabet Med 2002;19:70-3.

18. Chase HP, Garg SK, Cockerham RS, Wilcox WD, Walravens PA. Thyroid hormone replacement and growth of children with subclinical hypothyroidism and diabetes. Diabet Med 1990;7:299-303.

19. Chang CC, Huang CN, Chuang LM. Autoantibodies to thyroid peroxidase in patients with type 1 diabetes in Taiwan. Eur J Endocrinol 1998;139:44-8.

20. Nakazono M, Kudo M, Baba T, Kikuchi H, Takebe K. Thyroid abnormalities in diabetes mellitus. Tohoku J Exp Med 1983;141 Suppl:275-81.

21. Gebauer H, Pabst MA. Autoradiographic localization of $3 \mathrm{H}-\mathrm{GABA}$ uptake in the thyroid gland of the rat. Cell Tissue Res 1981;220:873-9.

22. Tsai LH, Taniyama K, Tanaka C. gamma-Aminobutyric acid stimulates acid secretion from the isolated guinea pig stomach. Am J Physiol 1987;253(5 Pt 1):G601-6. 